[Vicino Oriente XXIV (2020), pp. 85-107]

\title{
LE CERAMICHE D'IMPASTO DALLA ZONA D A MOZIA. ASPETTI SOCIO-ECONOMICI IN AMBITO DOMESTICO
}

\author{
Cecilia Guastella - Sapienza University of Rome
}

\begin{abstract}
This paper aims at examining the so-called Impasto Ware, discovered in the Area D of Motya, in order to identify and share information about the features of this class. Analyses of the vases section, of the shapes and diameters, and their technological features clarify the function (food preparation and cooking) and the production (primarly domestic and household) of IW. Some ethnographic parallels allow to define why the pottery-making process of $I W$ was being preserved in a long period in the community of Motya.
\end{abstract}

Keywords: Impasto Ware; cooking pottery; Elymian, Motya; household production

\section{PREMESSA}

Obiettivo di questo contributo è delineare le caratteristiche delle ceramiche di impasto rinvenute nella Zona D a Mozia ( $\S 3.)^{1}$, avvalendomi anche dell'expertise conseguita attraverso la mia ricerca di dottorato ${ }^{2}$.

La ceramica d'impasto o Impasto Ware (IW), attestata nei contesti indigeni, fenici e punici (§ 2.) della Sicilia, è prodotta per lo più a livello domestico. Essa è legata alla preparazione, alla cottura e al consumo degli alimenti ma è impiegata non di rado anche in ambito cerimoniale e funerario ${ }^{3}$. Le forme tipiche sono le pignatte, le teglie e gli scodelloni, a cui si aggiungono piastre, fornelli e sostegni a clessidra, spesso in associazione tra loro, costituendo un set tradizionale ${ }^{4}$. Il repertorio soffre della carenza di una documentazione omogenea per i numerosi siti di rinvenimento. Tuttavia, la maggiore conoscenza dei contesti e una rinnovata attenzione verso questa classe ceramica favoriscono oggi l'incremento del suo studio, in connessione con i contesti e con i repertori ceramici cronologicamente affidabili.

Benché sia definibile una specifica produzione locale, i repertori di IW in Magna Grecia, nel Vicino Oriente e in Africa settentrionale sono tra loro molto simili ${ }^{5}$.

Le analisi archeometriche costituiscono degli strumenti necessari ad integrare le informazioni deducibili dall'analisi della morfologia dei vasi, poichè forniscono dati aggiuntivi sull'intero processo tecnologico di produzione, dall'individuazione delle cave di

Già edito in Caltabiano - Spagnoli 2010 e Nigro (a cura di) 2007.

2 Il progetto di ricerca del dottorato, discusso presso 1'Università La Sapienza di Roma, è intitolato "Le ceramiche di impasto di tradizione locale, fenicia e punica in Sicilia", ed è stato condotto con il tutoraggio del Prof. Lorenzo Nigro. Sono in corso analisi chimico-fisiche dei contenuti dei vasi, al fine di evidenziare le correlazioni tra contenitore e contenuto. Tra i contesti oggetto di studio, il caso studio moziese riguarda direttamente le ceramiche provenienti dagli strati del tofet, indagati durante gli scavi diretti da Antonia Ciasca dal 1964 al 1973 Colgo l'occasione per ringraziare Federica Spagnoli, Chiara Iacopino e Nicola Chiarenza per le utili indicazioni. $\S 2$., nota 18 .

4 Orsingher 2013, 757-790; Spatafora 2000a, 895-918; Spatafora 2010a, 34-46; Russenberger 2010, 12-22; Di Stefano 2009; Valentino 2003, 255-267; Spanò Giammellaro 2000, 295-335.

5 Per un excursus sulle produzioni omonime nel Mediterraneo orientale e occidentale dei primi secoli del I millennio a.C., Spanò Giammellaro 2000, 295-335; Mansel 1999, 220-238; Torres - Maraouni Telmini 2011,153-165; Bernick Greenberg 2007, 131-186; Leighton ed. 1993; Leighton 2011, 197-214; Leighton Thompson 2012, 200-205.

ISSN 0393-0300

e-ISSN 2532-5159

Rivista Open Access 
argilla fino alle modalità di cottura e sugli usi del vaso. Per questo ultimo aspetto, cioè la determinazione della funzione, possono essere di grande utilità i confronti etnografici e le riproduzioni sperimentali $(\S 2 .)^{6}$. Lo studio delle ceramiche da fuoco, da preparazione e da mensa deve essere accompagnato dallo studio delle abitudini alimentari e delle tradizioni culinarie.

\section{INQUADRAMENTO DELLA PRODUZIONE DI IMPASTO IN SICILIA NELL'ETÀ DEL FERRO}

La ceramica di impasto $(\mathrm{IW})^{7}$ in Sicilia è diffusa nei contesti indigeni dalla fine del IX secolo a.C. e, in quelli fenici, dall'inizio dell'VIII secolo a.C. ma con maggiore intensità tra la seconda metà del VII e la prima metà del VI secolo a.C. La produzione continua fino alla prima metà del IV secolo a.C., quando si assiste ad una sua progressiva diminuzione ma non alla sua scomparsa, in particolare a Selinunte, a Monte Iato e a Monte Adranone ${ }^{8}$.

$\mathrm{La} \mathrm{IW}^{9}$ è tradizionalmente considerata una produzione locale, circoscritta ai confini dell'area occidentale della Sicilia, più precisamente nel territorio abitato dagli Elimi ${ }^{10}$ dall'inizio del I millennio a.C. ${ }^{11}$. A Mozia le prime attestazioni di IW sono databili già all'inizio dell'VIII secolo a.C. (Building C $8^{12}$ ) e sono ampiamente diffuse fino alla metà del IV secolo a.C. ${ }^{13}$. Al contrario sono assenti attestazioni nei livelli riferibili al Medio Bronzo nella Zona E, riconducibili all'orizzonte Thapsos-Milazzese (XV-XIV secolo a.C.) ${ }^{14}$. Nel repertorio moziese del $\mathrm{BM}$, infatti, le teglie, sebbene siano simili per forma a quelle del repertorio dell'IW (fig. 1), tuttavia presentano dei tipici e funzionali forellini sotto l'orlo che invece non caratterizzano le teglie più tarde (IW). Va inoltre rimarcato che il tipo di impasto dei contenitori del Thapsos-Milazzese è del tutto differente rispetto a quello dell'IW, poiché ha un maggiore peso specifico e un colore grigio o rosso bruno legato a una cottura a

6 Sulle produzioni domestiche, sulle maintenance activities e sulle cucine coloniali nei siti fenici e punici, Ferrer 2016, 11-31; Rice 1987; Santoro Bianchi - Guiducci - Tusa 2003; Cuomo di Caprio 2007; Skibo - Shiffer 2008 London 2016; Notarstefano 2012; Spataro - Villing 2015. Sulla Sardegna, Guirguis 2019, 111-126. Sui siti di Mozia e Cerro del Villar, Delgado - Ferrer 2007, 18-42.

7 Abbr. IW, Impasto Ware.

8 A Selinunte la IW è attestata nell'aerea sacra a Ovest del Tempio O, L.1048 e L.1050, Chiarenza 2019, 27-63; Chiarenza 2018, 278-284. Su Monte Iato si veda Russenberger 2010, 12-22. Su Monte Adranone si rimanda ad alcune considerazioni preliminari in Guastella 2018, 86-87.

9 Nigro 2013, 39-74; Spatafora 2010a, 34-46; Spanò Giammellaro 2000, 295-335; Guastella 2018, 81-96.

10 La questione è attestata nella storiografia classica, Tucidide, Thuc, VI, 2, 3; Ellanico, FGrHist 4 F 79b. Si rimanda a Nenci - Tusa S. - Tusa V. 1990; Spatafora - Sciortino 2015, 221-229; Spatafora 2010a, 34-46; Tusa 2015, 289-309; Tusa 1990, 31-54; Spatafora - Vassallo 2005; Bondì 2002, 87-94. Negli ultimi studi le interpretazioni sui processi storici della Sicilia superano la cesura tra le aree orientale e occidentale dell'isola, prima considerate in maniera distinta. Emergono nella parte occidentale dell'isola apporti culturali dalla Sicilia orientale sicana e dalle coste verso l'entroterra.

11 Ceramiche incise, impresse a cerchielli, dipinte, le tipiche anse sopraelevate con protome antropo-zoomorfe (Monte Finestrelle) indicano il sopraggiungere di una nuova produzione artigianale. Nelle fasi di VIII secolo a.C. la realtà locale è ricca di differenti tratti culturali, sicani (S. Angelo Muxaro e Polizzello), pensinsulari e orientali. In Sardegna si assiste a un processo simile già dal Bronzo Finale e poi, più intensamente, dagli ultimi decenni del IX secolo a.C., Bernardini 2000, 29-61; Bernardini 2014, 73-87; Guirguis 2012; Usai 2013, 23-34 Sulla ceramica fatta a mano e la definizione dell'entità "indigena" in stanziamenti fenici in Sardegna dell'età del Ferro I e II, Usai 2013, 26

12 Nigro 2013, 39-74; Nigro - Spagnoli 2017, 33-34.

13 Orsingher 2011, 112-131.

14 Spatafora 2000b, 919-956; Famà - Toti 2000, 451-478; Guastella 2018, 88-89; Nigro 2016, 347-370. 
temperatura più elevata ${ }^{15}$. Nella parte occidentale della Sicilia i confronti più vicini con produzioni di fasi protostoriche per la IW sono databili alla prima età del Ferro e si riferiscono alla tarda facies di S. Angelo Muxaro. Le ceramiche di questa facies, tuttavia, hanno impasti pesanti e compatti, non presentano standardizzazione della produzione delle tre forme canoniche, pignatta, teglia e scodellone, tipica della IW, e non includono la tipica forma della pignatta tetransata a fondo piatto. Per le sue caratteristiche morfologiche e tecnologiche, la IW in oggetto sembra essere più vicina alle produzioni in impasto levantine e nord-africane ${ }^{16}$. Una delle caratteristiche generali della IW rinvenuta in Sicilia è la presenza di tipologie e dimensioni standardizzate di pignatte, teglie e scodelloni, collegate al consumo di pietanze tipiche, come zuppe a base di cereali, legumi e carne ${ }^{17}$. Inizialmente diffuse in contesti domestici e in luoghi di culto ${ }^{18}$, dal VII secolo a.C. esse sono presenti nei corredi funerari a Mozia e a Palermo ${ }^{19}$. Tra la metà del VII e la metà del VI secolo a.C., le pignatte sono impiegate come urne nel tofet di Mozia: in questo periodo, infatti, il rapporto tra i Fenici e la comunità indigena era solido tanto da far supporre che il processo di integrazione fosse ormai completo $^{20}$. La presenza nelle tombe di vasi così fortemente legati ad un utilizzo quotidiano e, probabilmente, ad una specifica tradizione culinaria, se non a un particolare alimento, doveva avere nell'antichità un significato chiaro per i contemporanei. In base ai dati a nostra disposizione, possiamo ipotizzare che questo fosse legato all'appartenenza a un gruppo sociale o ad una particolare categoria e quindi dovesse essere portatore di una qualche valenza identitaria $^{21}$. L'affiancarsi dei vasi di impasto nei corredi funerari e nel tofet, alle tipiche forme di tradizione fenicia, tra cui le olle monoansate, indica una condivisione delle pratiche religiose e funerarie ${ }^{22}$.

15 Spatafora 2000b, 926, tav. CLXXX.

16 Sulle caratteristiche della IW moziese e siciliana: $§ 1$., nota 4. Sui confronti con produzioni della Sicilia orientale e con la Transgiordania e il Nord Africa, Spanò Giammellaro 2000, 307; Guastella 2018, 82, note 17-22 e 91.

17 Spanò Giammellaro 2004a, 417-464; Nigro 2013, 39-74; Orsingher 2013, 757-790; Guastella 2018, 81-96.

18 La forma è attestata a Mozia nei corredi funerari già nella Fase 10, Motya IVB (750-675 a.C.), ad esempio nella T.164, dove la pignatta è associata a un'anfora IW con collo a tromba, Nigro - Spagnoli 2017, 66.

19 Si rimanda all'ampia bibliografia sulle indagini della necropoli e del tofet a Mozia seguite da Antonia Ciasca. Inoltre, Spatafora 2010a, 34-46; Spatafora 2012, 59-90; Spatafora ed. 2015; Vecchio 2013, 43-67; Aubet 2010, 13-22; Tusa 2008, 67-85; Spanò Giammellaro 2004a, 417-464; 2004b, 205-251; Delgado - Ferrer 2007, 18-42; Orsingher 2013, 757-790; Orsingher 2018, 29-34. Sulla necropoli punica di Palermo Di Stefano 2009. Nelle tombe a fossa con rito a incinerazione della necropoli punica di Palermo sono frequenti le pignatte nei corredi, con repertori tipicamente punici di fine VII - prima metà VI secolo a.C., Spatafora 2012, 66-68.

20 Spanò Giammellaro 2004b, 205-251; Spatafora 2010b, 25-39; Nigro 2015, 225-245.

21 Spanò Giammellaro 2004a, 417-464.

22 Questo aspetto collega l'uso di forme specifiche agli usi alimentari, funerari e ai riti diffusi nel Mediterraneo durante la presenza fenicia e punica, Delgado - Ferrer 2011, 184-203; Delgado 2008, 163-188; Delgado 2010 , 33-52; Campanella 2008; Niveau - Castro 2008, 7-48. Sugli studi nell'area cananea, Oggiano 2005; Spagnoli 2010 . 


\section{IL REPERTORIO CERAMICO DI IMPASTO NELLA ZONA D}

Giueppe Whitaker indagò per primo la zona situata alle pendici occidentali dell'Acropoli di Mozia (fig. 2) e ne identificò la destinazione ad uso abitativo ${ }^{23}$. A partire dal 2002, la Missione Archeologica della Sapienza Università di Roma, in collaborazione con la Soprintendenza per i Beni Culturali e Ambientali di Trapani e con la Fondazione Whitaker, ha intrapreso le indagini nella Zona D.

L'occupazione di questo settore dell'isola copre un arco temporale che va dalla fine del Bronzo Antico e l'inizio del Bronzo Medio (1500-1250 a.C.) ${ }^{24}$ (fig. 3), sino alla prima metà del IV secolo a.C. ${ }^{25}$. Le ceramiche di impasto della Zona D sono state rinvenute negli strati relativi a tre periodi: Motya IVB, metà dell'VIII - inizi del VII secolo a.C. (Fase 10) ${ }^{26}$; Motya VII, V secolo a.C. (Fase 5); Motya VIII, metà IV secolo a.C. (Fase 3) ${ }^{27}$. Tra l'VIII e il VI secolo a.C. si registra la maggiore presenza di IW. La collocazione di questi materiali all'interno di contesti cronologicamente affidabili consente di avere delle datazioni attendibili e di osservare i cambiamenti diacronici nelle tecniche di manifattura.

\subsection{Motya IVB (Fase 10)}

Alcune pignatte, datate tra la metà dell'VIII e gli inizi del VII secolo a.C., sono state ritrovate nelle colmate US/F.2246 e US/F.2219, realizzate a metà del VI secolo a.C. con materiali di VIII-VII secolo a.C. per pareggiare il pendio dell'acropoli quando l'area venne adibita a quartiere residenziale ${ }^{28}$. Altre pignatte con profilo troncoconico ${ }^{29}$, databili tra la metà dell'VIII e gli inizi del VII secolo a.C., sono state rinvenute nella colmata della fossa F.1112, realizzata nello stesso periodo poco più a sud ${ }^{30}$.

MD.07.2246/19 (figg. 4-5)

Pignatta troncoconica.

Provenienza: colmata US.2246.

Tecnica: a mano, colombino e a pressione.

Caratteristiche: $\varnothing 15 \mathrm{~cm}$. Matrice medio-grossolana, inclusi calcarei e vegetali. Orlo piatto superiormente introflesso, due prese a semiluna (particolari rispetto alle frequenti bugne o prese a linguetta e che trovano continuità fino al IV secolo a.C.). Superficie esterna lustrata, interna lisciata. Superficie esterna annerita, in particolare sulla presa, indice dell'esposizione diretta al fuoco.

Datazione: metà VII - inizi VI secolo a.C.

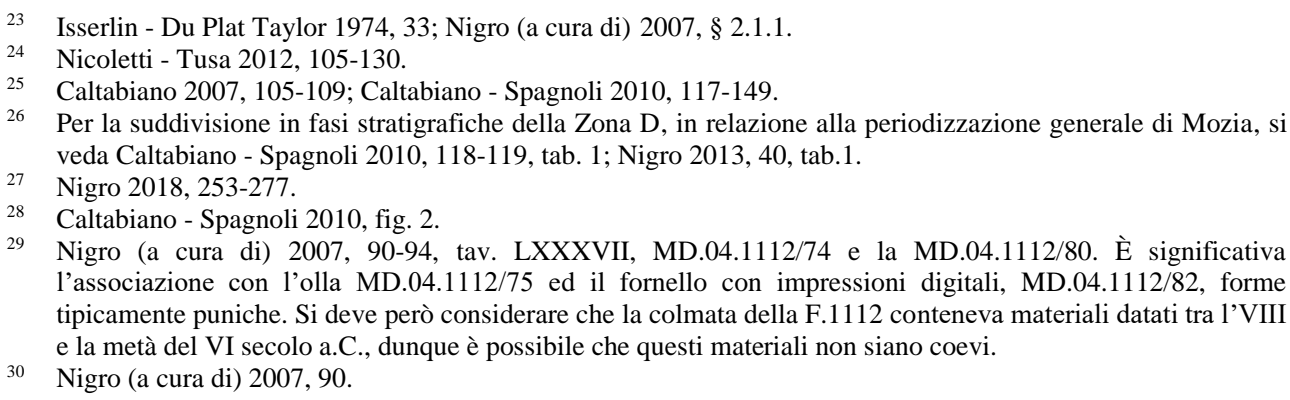


Confronti: Mozia, pignatta con presa ad orecchio, strato IV b del Cappiddazzu, metà VII inizi VI secolo a.C., Ciasca - Tusa - Uberti 1973, tav. X, fig. 1:d. Pignatta MF.04.1273/11, Locus 1224 Fortezza Occidentale, in US.1273, strato inferiore addossato sul pavimento di ceneri e carboni con materiali schiacciati dallo stesso crollo, Nigro (a cura di) 2007, 37, tav. LXXVII.

Bibliografia: Caltabiano - Spagnoli 2010, 130, tav. XIII.

MD.04.1112/74 (fig. 6)

Pignatta troncoconica.

Provenienza: F.1112.

Tecnica: a mano, a colombino.

Caratteristiche: matrice mediamente grossolana ma poco friabile, inclusi di chamotte, calcarei e vegetali in media quantità. Bugne molto sporgenti. Tracce di patina bruna omogenea sulla parete interna e annerimenti a chiazza sulla parete bassa esterna.

Datazione: metà VIII - inizi VII secolo a.C.

Bibliografia: Nigro (a cura di) 2007, 90-94, tav. LXXXVII.

MD.04.1112/80 (fig. 7)

Pignatta tronconica.

Provenienza: F.1112.

Tecnica: a mano, a colombino.

Caratteristiche: impasto mediamente depurato. Pareti sottili, presa a semiluna applicata orizzontalmente sulla parete, superfici lisciate.

Datazione: metà VIII - inizi VII secolo a.C.

Confronti: Monte Maranfusa, Valentino 2003, 256, fig. 221:10.

Bibliografia: Nigro (a cura di) 2007, 90-94, tav. LXXXVII.

\subsection{Motya VII (Fase 5)}

La ceramica di impasto di questo periodo, ascrivibile al V secolo a.C., è stata identificata nella Casa del Sacello domestico, una residenza a corte centrale provvista di una serie di vani $^{31}$.

\section{MD.03.1009/12 (fig. 8)}

Pignatta troncoconica.

Provenienza: corte L.220.

Tecnica: mista, a mano e al tornio lento.

Caratteristiche: matrice medio-grossolana, inclusi calcarei. Bugne, pareti interne ed esterne lisciate con un panno.

Datazione: V secolo a.C.

Bibliografia: Nigro (a cura di) 2007, 14, 94, tav. XXXV.

MD.04.1042/9 (figg. 14-15)

Scodellone.

Provenienza: strato di copertura del magazzino L.266, Casa del Sacello domestico.

Tecnica: a mano, a pressione.

31 Nigro (a cura di) 2007. 
Caratteristiche: $\varnothing 32 \mathrm{~cm}$. Impasto friabile, alta quantità di degrassanti vegetali e minerali di piccole e medie dimensioni. Superficie interna ed esterna lisciata in senso orizzontale. Corpo ceramico scuro, superficie interna annerita sotto l'orlo, è evidente la traccia del livello del liquido contenuto (fig. 15).

Datazione: fine V secolo a.C.

Bibliografia: Nigro (a cura di) 2007, 54, tav. XXV.

MD.03.1042/13 (fig. 16)

Scodellone.

Provenienza: strato di copertura del magazzino L.266, Casa del Sacello domestico.

Tecnica: a mano, colombino e a pressione.

Caratteristiche: $\varnothing$ non rilevabile. Impasto mediamente grossolano, media quantità di inclusi minerali. Simile ad un tegame, con anse a bastoncello impostate orizzontalmente e risega per l'alloggio del coperchio estranea alla produzione.

Datazione: fine V secolo a.C.

Confronti: Mozia, Nigro (a cura di) 2005, tav. LXXXVII, scodellone MC.04.710/11, strato di crollo del Tempio C2 nel Kothon (fig. 17). Morgantina, scodellone di fine V secolo a.C., Leighton ed. 1993, tav. 49:469.

Bibliografia: Nigro (a cura di) 2007, tav. LIV.

MD.04.266/8 (fig. 18)

Fornello.

Provenienza: strato di copertura del magazzino L.266, Casa del Sacello domestico.

Tecnica: a mano.

Caratteristiche: $\varnothing 16 \mathrm{~cm}$. Impasto grossolano, ricco di inclusi vegetali. Incisioni lineari parallele che si sviluppano in senso verticale sulla parete esterna ed interna.

Datazione: VI - V secolo a.C.

Confronti: tipo IC classificazione Scheffer, diffuso nel VI secolo a.C. nell'Italia centromeridionale, Scheffer 1981, 73; Campanella 2009, 469-496.

Bibliografia: Nigro (a cura di) 2007, 55, tav. XXX.

\subsection{Motya VIII (Fase 3)}

Ceramica di tradizione punica e locale, per lo più da cucina, proviene dal locus L.240 e dallo strato di crollo US.216 nella Casa del Sacello domestico, entrambi databili alla metà IV secolo a.C. ${ }^{32}$.

\section{MD.02.216/16 (fig. 9)}

Pignatta troncoconica.

Provenienza: strato di copertura del magazzino L.266, Casa del Sacello domestico.

Produzione: a mano; a colombino e a pressione.

Caratteristiche: $\varnothing 16 \mathrm{~cm}$. Orlo introflesso e assottigliato $(0,6 \mathrm{~cm})$, pareti rastremate verso l'alto. Quattro prese ad orecchio a sezione rettangolare, impostate sotto l'orlo. Superfici lisciate. Tracce di una patina grigia sulla parete interna, al di sotto dell'orlo, riferibile alla bollitura dell'alimento contenuto.

32 Nigro (a cura di) 2004, 155, tav. LX 
Confronti: metà IV secolo a.C.

Bibliografia: Nigro (a cura di) 2004, 155, tav. LX.

MD.02.216/48 (figg. 10-11)

Teglia.

Provenienza: vano L.240, Casa del Sacello domestico.

Tecnica: a mano, a pressione.

Caratteristiche: $\varnothing 22 \mathrm{~cm}$. Fondo piatto realizzato a mano con tecnica a pressione. Il vasaio ha reso il fondo largo e spesso e con maggiore spessore ai lati, garantendo maggiore robustezza. La parete è stata secondariamente inspessita. Il vasaio scavava dall'interno partendo da un nucleo di argilla, aggiungendo solo in seguito eventuali colombini (fig. 11). Il punto di giuntura tra fondo e la parete è caratterizzato da una modanatura smussata che armonizzava il profilo e favoriva la resistenza al forte calore. Fondo interno e parte del corpo ceramico sono anneriti, a seguito della cottura dei cibi ${ }^{33}$.

Datazione: metà IV secolo a.C. ${ }^{34}$.

Bibliografia: Nigro (a cura di) 2004, 155, tav. LX.

MD.04.1090/15 (figg. 12-13)

Teglia.

Provenienza: strato di superficie US 1090, Casa del Sacello domestico.

Produzione: a mano, a pressione.

Caratteristiche: $\varnothing 36 \mathrm{~cm}$. Impasto grossolano, alta presenza di inclusi vegetali e minerali. Superficie esterna ed interna lisciate, in senso verticale (fig. 13). Tracce di annerimento nel corpo ceramico e nella parete interna sotto l'orlo, dovute alla poca ossigenazione durante la cottura.

Datazione: metà IV secolo a.C.

Confronti: Monte Maranfusa, Valentino 2003, 263, fig. 225:49; Spatafora 2003, 99, fig. 114:43. Mozia, teglia MC.04.982/18, dal piano di calpestio L.982 dell'adyton del tempio C2, databile al V secolo a.C., Nigro (a cura di) 2005, 103-104, tav. CXXVIII. Caltabiano Spagnoli 2010, 119.

Bibliografia: Nigro (a cura di) 2007, 11, tav. LXIV.

\section{LA CERAMICA DI IMPASTO A MOZIA: ASPETTI TECNOLOGICI E CICLO PRODUTTIVO}

Nel gruppo delle ceramiche di impasto della Zona D (fig. 19) ricorrono tre forme: le pignatte, prevalentemente con profilo troncoconico con diametro dell'orlo compreso tra i 15 e i $16 \mathrm{~cm}$, e le teglie e gli scodelloni con un diametro di $30 \mathrm{~cm}$ circa $^{35}$. La capienza dei contenitori sembra corrispondere alla razione di un nucleo familiare di 6-8 persone ${ }^{36}$. Nella IW moziese la matrice dell'impasto ha una tessitura grossolana e una consistenza da molto friabile a poco compatta. Il tipo di argilla è di provenienza locale, come si evince dal colore ${ }^{37}$ e dagli inclusi calcarei e quarzosi, ai quali sono aggiunti, come degrassanti, inclusi vegetali

Caltabiano - Spagnoli 2010, 119.

34 Frammenti di queste teglie erano presenti nell'US.2206, corrispondente al crollo del piano superiore collassato dell'edificio D5, di VII secolo a.C. (Nigro (a cura di) 2004, 155, tav. LX).

35 Per il calcolo approssimativo dei diametri si è considerato un numero di individui (NMI) su base qualitativa, Orton - Tyers - Vince 1993, 166-181.

36 Skibo - Scheffer 1995, 80-91; Skibo - Scheffer 2008; Skibo 1992

37 5YR5/6 Yellowish Red, 5YR7/6 Reddish Yellow e 10R4/6 Red. 
(come la paglia) e la chamotte. L'impasto ricco di inclusi obbliga alla lavorazione a mano: l'alta concentrazione di inclusi di grandi e medie dimensioni rende, infatti, la superficie poco coesa e particolarmente difficile da lavorare al tornio, anche quando la tecnica del tornio è ampiamente attestata e utilizzata ${ }^{38}$. Questi contenitori sono prodotti per essere esposti alle alte temperature e al fuoco diretto, garantendo la resistenza agli shock termici. L'alta presenza di inclusi, gli spigoli smussati e le pareti rastremate verso l'alto ne determinano la resistenza e una buona conduttività del calore (in particolare si veda la pignatta MD.02.216/16). Una produzione con queste caratteristiche dipende dall'abilità del vasaio che dispone delle argille locali con i degrassanti naturalmente presenti, ai quali aggiungere elementi minerali e vegetali in quantità opportuna.

Le ceramiche sono realizzate a mano, in un solo caso è possibile riconoscere l'uso del tornio lento (MD.03.1009/12). In generale, per realizzare questi vasi, si utilizzava una tecnica mista, a pressione e a colombino, come mostrano le giunture semplici e le immorsature di colombini e/o fascette, poste orizzontalmente lungo la parete del vaso, visibili in sezione ${ }^{39}$.

I vasi in IW della Zona D sono tutti sottoposti a trattamenti superficiali, lisciatura e lucidatura della superficie, e una particolare cura è riservata alla parete interna dove è necessario garantire l'impermeabilità del contenitore. Il vasaio, con un movimento ripetitivo in senso orizzontale, utilizzava polpastrelli, pezzuole e/o lisciatoi (ciottoli o bastoncelli), dal momento in cui l'argilla aveva la consistenza "cuoio", ottenendo così la cosiddetta steccatura. Il trattamento superficiale era applicato con più insistenza all'interno del vaso e sull'orlo, mentre l'esterno poteva essere risparmiato del tutto e questo rendeva il contenitore più vulnerabile agli urti e alle fratture. Un'ultima considerazione riguarda la cottura: i contenitori rinvenuti nella Zona D hanno un colore variabile rosso-arancio, tipico dell'argilla locale, che indica anche una cottura in forni domestici con atmosfera ossidante. Probabilmente i vasi erano cotti coperti con del combustibile (rami secchi, sterpaglia, paglia, legna ${ }^{40}$ ) che inizialmente favoriva una camera d'aria riducente; successivamente il passaggio di ossigeno aumentava sufficientemente in modo da consentirne un buon ricircolo. La temperatura non doveva superare i $750^{\circ}$. Questo limite è confermato sia dalla morbidezza delle argille, che sono molto friabili, sia dalla presenza abbondante di calcite che, ad una maggiore temperatura di esposizione, decomponendosi avrebbe determinato rischi di rottura ${ }^{41}$.

\section{CONFRONTI ETNOGRAFICI}

I confronti etnografici con alcune produzioni ceramiche domestiche contemporanee, seppur prodotte in contesti lontani, cronologicamente e geograficamente, da quelli presi in esame, sono utili nel tentativo di ricostruire aspetti della produzione della ceramica d'impasto a Mozia, anch'essa riconducibile a un contesto domestico e familiare. In villaggi in Africa, nella costa settentrionale della Tunisia, a Sud-Ovest del continente, nel Burkina Faso, infine, spingendosi fino al Sud-Est asiatico nelle Filippine, ancora oggi le donne tramandano la tradizionale produzione a mano di ceramiche da fuoco da una generazione a un'altra, da

Santoro Bianchi - Guiducci - Tusa 2003, 58

Cuomo di Caprio 2007; Levi 2010; Halstead 2012, 21-51; Delgado - Ferrer 2007, 18-42.

Cuomo di Caprio 2007, 505; Spanò Giammellaro 2004a, 431-432.

1 Bettelli - Levi 2003, 440. 
madre a figlia, da nonna a nipote, assumendo così un intimo significato affettivo ${ }^{42}$. La preferenza della produzione a mano persiste da secoli se non da millenni e costituisce ancora oggi una scelta in molti territori del Mediterraneo ${ }^{43}$.

In Tunisia, a Ovest dalla città di Bizerte, si trova il villaggio di Sejnane, dove le tipiche ceramiche da fuoco e da mensa sono realizzate con la tecnica a colombino dalle donne, uniche depositarie delle tecniche di produzione, definite di antichissima tradizione ${ }^{44}$. La ceramica di Sejnane costituisce un confronto interessante, trovandosi sull'altra sponda del Mediterraneo rispetto all'isola di Mozia. La produzione di pignatte e di teglie, di simile fattura rispetto a quelle oggetto di studio, si snoda per un tempo lunghissimo, dall'VIII secolo a.C. fino al X secolo d.C. Ancora oggi, le ceramiche da fuoco di Sejnane sono regolarmente utilizzate nelle case della zona e sono espressione del patrimonio culturale della comunità ${ }^{45}$. Una realtà affine si ritrova nelle Filippine, nel villaggio di Kalinga; anche in questo caso, sono le donne a tramandare la tipica produzione di olle, utilizzate principalmente per la cottura del riso, pietanza base della dieta quotidiana locale. I vasi sono ancora volontariamente prodotti in contesti domestici, a mano e in argilla, preferita al metallo perché garantisce una cottura migliore degli alimenti. Le misure dei contenitori sono standardizzate, sulla base dei consumi da parte del nucleo familiare. Le olle sono utilizzate anche per eventi rituali e religiosi, matrimoni o funerali, in vista dei quali sono conservate con cura, lontano dal focolare (fig. $20)^{46}$.

Anche a Mozia la IW doveva essere legata al mondo femminile: proprio le donne autoctone, tramandavano l'uso di questi contenitori e verosimilmente introdussero a Mozia le abitudini alimentari diffuse nella Sicilia occidentale. L'impiego delle ceramiche di impasto nel tofet e nella necropoli è un ulteriore espressione del significato identitario e, associate a ceramiche di tradizione levantina, riflettono la composizione variegata e coesa della comunità moziese. Nella Casa del Sacello domestico la compresenza di ceramiche da fuoco tornite, di tradizione fenicia, e ceramiche di impasto, spinge a pensare che si volesse mantenere volontariamente l'uso di vasi in IW per ragioni funzionali ${ }^{47}$ (così come avviene nel villaggio di Kalinga, dove si continua a preferire l'olla in argilla a quella in metallo) e per ragioni culturali, nella misura in cui il processo produttivo invariato diviene elemento di riconoscibilità e di continuità con la tradizione.

42 Skibo - Schiffer 1995, 83-84; Skibo - Schiffer 2008, 109-111.

43 Sull'argomento si rimanda a London 2016, in particolare all' excursus a partire dal Neolitico sulla produzione ceramica da cucina e alle pratiche corredate a Cipro e nel Levante, dove in alcuni abitati, la ceramica da fuoco viene realizzata ancora oggi a mano. Si veda Camps 1980( 282-285) sulle possibili dinamiche di diffusione delle tecniche di produzione tra Africa settentrionale, Sicilia e Italia peninsulare.

44 Sekik 2007. Le produzioni medievali di Sejinane sono affini alle produzioni medievali della Sicilia di X - XIII secolo d.C., Spanò Giammellaro 2000, n. 79; Guastella 2018, 81-96.

45 Camps 1980, 282-285; Sekik 2010, 29-35. La produzione ceramica di Sejinane dal 2018 è stata definita patrimonio culturale immateriale dell'umanità dall'UNESCO.

46 Skibo 1992, 60

47 Spanò Giammellaro 2004a, 417-464. 


\section{CONCLUSIONI: ASPETTI ECONOMICI E IMPLICAZIONI SOCIALI DELLA PRODUZIONE DI IMPASTO A MOZIA}

Il repertorio delle ceramiche d'impasto della Zona D permette di fare alcune considerazioni. La persistenza di alcune caratteristiche morfologiche e tecnologiche suggerisce una produzione tradizionale e domestica destinata al nucleo familiare. Durante il VII e il VI secolo a.C., periodo al seguito del quale si diffonde l'impiego del tornio veloce, la manifattura di questa classe ceramica è verosimilmente femminile, indipendente da una produzione a livello industriale; si riconoscono, piuttosto, le caratteristiche di una household production $^{48}$. L'analisi dei contesti abitativi consente pertanto di ricostruire una catena produttiva mirata al soddisfacimento delle esigenze alimentari del nucleo familiare ${ }^{49}$ L'interazione delle tecniche è il risultato di un sapere artigianale "tacito" che sfrutta materie e strumenti a basso costo, in un ambiente informale come quello domestico ${ }^{50}$. L'ampiezza dell'arco temporale, in cui le fogge e le tecniche di realizzazione rimangono invariate, evidenzia la valenza sociale di questa produzione e la sua importanza economica all'interno della comunità. Ad esso si lega un sistema alimentare tradizionale, concepito come veicolo di scambio culturale e di auto-rappresentazione di insiemi eterogenei uniti dalla percezione dell'appartenenza a un comune gruppo sociale.

\section{BIBLIOGRAFIA}

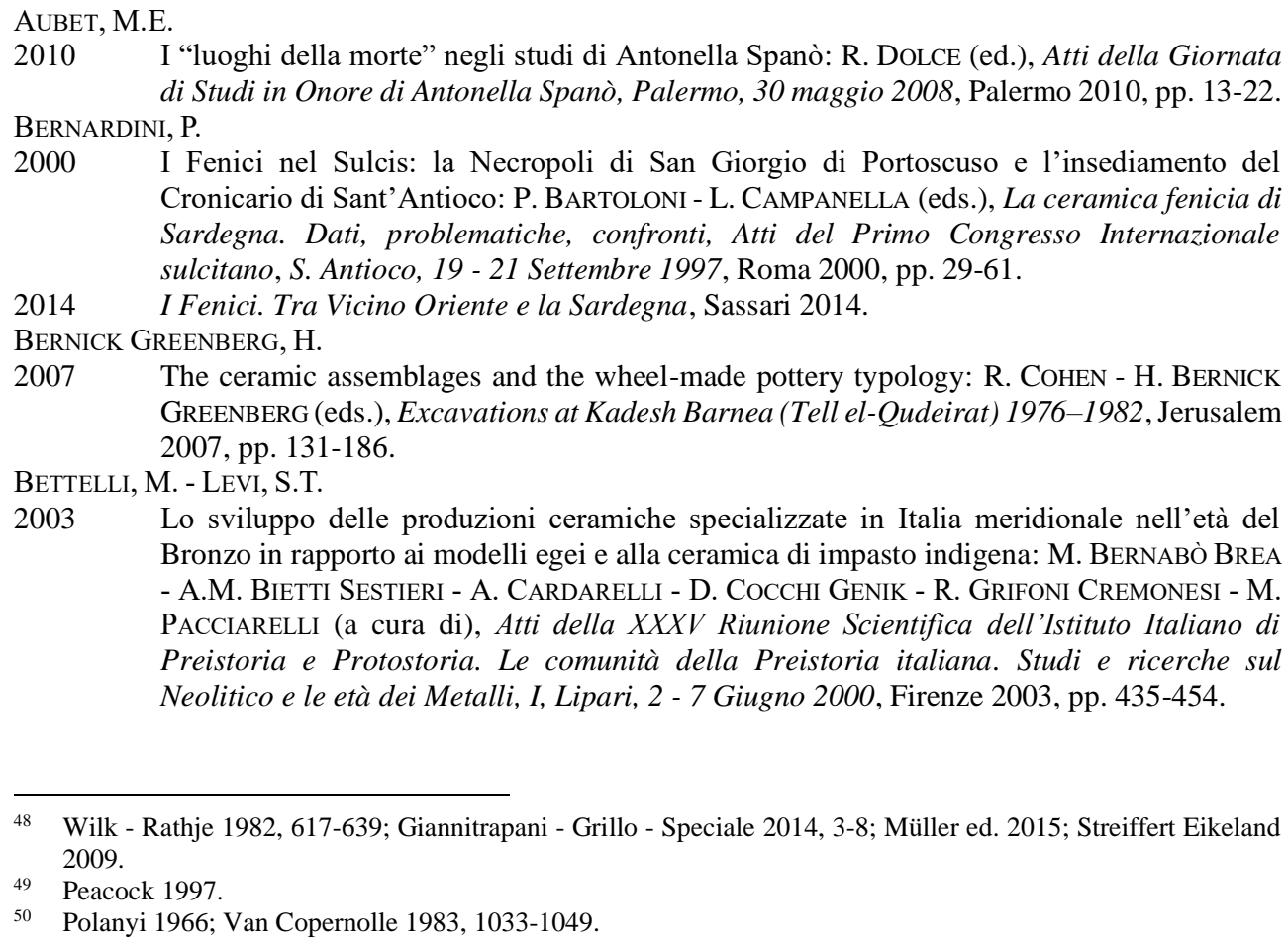

2000 I Fenici nel Sulcis: la Necropoli di San Giorgio di Portoscuso e l'insediamento del Cronicario di Sant'Antioco: P. BARTOLONI - L. CAMPANELla (eds.), La ceramica fenicia di Sardegna. Dati, problematiche, confronti, Atti del Primo Congresso Internazionale sulcitano, S. Antioco, 19 - 21 Settembre 1997, Roma 2000, pp. 29-61.

$2014 \quad$ I Fenici. Tra Vicino Oriente e la Sardegna, Sassari 2014.

BERNICK GREENBERG, H.

2007 The ceramic assemblages and the wheel-made pottery typology: R. COHEN - H. BERNICK GREENBERG (eds.), Excavations at Kadesh Barnea (Tell el-Qudeirat) 1976-1982, Jerusalem 2007, pp. 131-186.

BetTelli, M. - LEVI, S.T.

2003 Lo sviluppo delle produzioni ceramiche specializzate in Italia meridionale nell'età del Bronzo in rapporto ai modelli egei e alla ceramica di impasto indigena: M. BERNABÒ BREA - A.M. BietTi Sestieri - A. CARDARelli - D. Cocchi GeniK - R. Grifoni CRemONESI - M. Pacciarelli (a cura di), Atti della XXXV Riunione Scientifica dell'Istituto Italiano di Preistoria e Protostoria. Le comunità della Preistoria italiana. Studi e ricerche sul Neolitico e le età dei Metalli, I, Lipari, 2 - 7 Giugno 2000, Firenze 2003, pp. 435-454.

48 Wilk - Rathje 1982, 617-639; Giannitrapani - Grillo - Speciale 2014, 3-8; Müller ed. 2015; Streiffert Eikeland 2009.

49 Peacock 1997.

50 Polanyi 1966; Van Copernolle 1983, 1033-1049. 
BONDÍ, S.F.

2002 Dalle città ai comprensori: prospettive recenti sulla Sicilia fenicia e punica: P. DonATI GiaComini - M.L. Uberti (edd.), Fra Cartagine e Roma. Seminario di studi italo-tunisino, Bologna, 23 Febbraio 2001, Faenza 2002, pp. 87-94.

Caltabiano, A.

2007 La ceramica preistorica del Sondaggio I: L. Nigro (ed.), Mozia, Zona D, la "Casa del sacello domestico", il "Basamento meridionale" e il Sondaggio stratigrafico I. Rapporto preliminare delle campagne di scavi XXIII e XXIV (2003-2004) condotte congiuntamente con il Servizio Beni Archeologici della Soprintendenza Regionale per i Beni Culturali e Ambientali di Trapani (Quaderni di Archeologia Fenicio-Punica III), Roma 2007, pp. 105 109.

Caltabiano, A. - Spagnoli, F.

2010 Mozia: la ceramica fenicia arcaica dal sondaggio stratigrafico III nella Zona D: L. NIGRO (ed.), Motya and the Phoenician ceramic repertoire between the Levant and the West - 9th6th Century B.C. Proceedings of the International Conference Held in Rome (Quaderni di Archeologia Fenicio Punica V), Roma 2010, pp. 117-149.

CAMPANELla, L.

2008 Il cibo nel mondo fenicio e punico d'Occidente: un'indagine sulle abitudini alimentari attraverso l'analisi di un deposito urbano di Sulky in Sardegna (Collezione Studi Fenici 43), Pisa 2008.

2009 I forni, i fornelli e i bracieri fenici e punici: J. BONETTO - G. FALEZZA - A.R. GHIOTTO (edd.), Nora. Il Foro Romano. Storia di un'area urbana dall'età fenicia alla tarda antichità. I

CAMPS, G. materiali pre-romani, II.1, Padova 2009, pp. 469-496.

$1980 \quad$ Les Berbères. Mémoire et Identité, Parigi 1980.

CHIARENZA, N.

2018 All'ombra delle colonne: le aree sacre ai margini del Tempio O nella Selinunte punica: M. Guirguis (ed.), From the Mediterranean to the Atlantic: people, goods and ideas between East and West. II. 8th International Congress of Phoenician and Punic Studies, Sardinia Carbonia, Sant'Antioco 21th-26th October 2013 (Folia Phoenicia 2), Pisa - Roma 2018, pp. 278-284.

2019 Selinunte tra la seconda metà del IV e il III secolo a.C. Un insediamento dell'eparchia cartaginese al centro del Mediterraneo: Karthago revue d'histoire et d'archéologie africaines 31 (2019), pp. 27-63.

Ciasca, A. - Tusa, V. - Uberti, M.L.

1973 Mozia VIII. Rapporto preliminare della Missione congiunta con la Soprintendenza alle Antichità della Sicilia occidentale (Studi Semitici 45), Roma 1973.

CUOMO DI CAPRIO, $\mathrm{N}$.

2007 Ceramica in archeologia, 2: antiche tecniche di lavorazione e moderni metodi di indagine, Roma 2007

Delgado Hervás, A.

2008 Alimentos, poder e identidad en las comunitas fenicias occidentales: Cuadernos de Preistoria y Arquelogìa de la Universidad de Granada 18 (2008), pp. 163-188.

2010 De las cocinas coloniales y otras historias silenciades, Saguntum, extra 9 (2010), pp. 33-52. Delgado Hervás, A. - Ferrer Martín, M.

2007 Cultural contacts in colonial settings: the construction of new identities in Phoenician Settlements of the Western Mediterranean: Stanford Journal of Archaeology 5 (2007), pp. $18-42$. 
2011 Representing communities in heterogeneous worlds: staple food and ritual practices in the Phoenician diaspora: G.A. JimÉnes - S. Montòn-SubìAS - M. SÁNCHES Romero (eds.), Guess Who's coming to dinner. Feasting rituals in the Prehistoric societies of Europe and the Near East, Oxford 2011, pp.184-203.

Di Stefano, C.A.

2009 La Necropoli punica di Palermo. Dieci anni di scavi nell'area della Caserma Tuköry, Pisa - Roma 2009

FAMÀ, M.L. - TOTI, M.P.

2000 Materiali dalla Zona E dell'Abitato di Mozia. Prime considerazioni: A. CorretTi (ed.) Giornate Internazionali di Studi sull'area elima, 3, Pisa - Gibellina, 23 - 26 Ottobre 1997, Pisa 2000, pp. 451-478.

FERRER, M.

2016 The forgotten things. Women, rituals, and community in Western Sicily (eight-sixth centuries BCE): M. DiLLON - E. EIDINOW - L. MAURIZIO (eds.), Women's Ritual Competence in the Greco-Roman Mediterranean, London - New York 2016, pp. 11-31.

Giannitrapani, E. - Grillo, F.M. - Speciale, C.

2014 Household archaeology nella Preistoria siciliana: A. SPOsito (a cura di), Agathòn. Recupero dei contesti antichi e processi innovativi dell'architettura (2014), pp. 3-8.

Guastella, C.

2018 Approaching to the impasto Ware of local, Phoenician and Punic tradition in Sicily: Sardinia, Corsica et Baleares Antiqvae XVI (2018), pp. 81-96.

GuIRGUIS, M.

2012 Tyrio fundata potenti. Temi sardi di archeologia fenicio-punica, Sassari 2012.

2019 Central North Africa and Sardinian Connections (end of 9th-8th century BC). The multiethnic and multicultural facies of the earliest western Phoenician communities: S. DI Lernia - M. Gallinaro (eds.), Arid Zone Archaeology. Archaeology in Africa. Potentials HALSTEAD, P. and perspectives on laboratory \& fieldwork research 8 (2019), pp. 111-126.

2012 Feast, food and fodder in Neolithic-Bronze Age Greece: commensality and the construction of value: S. Pollock (ed.), Between Feasts and Daily Meals: Toward an Archaeology of commensal spaces, Berlin 2012, pp. 21-51.

Isserlin, B.S.J. - Du Plat TAYloR, J.

1974 Motya. A Phoenician and Carthaginian City in Sicily. A report of the excavations undertaken during the years 1961-65 on behalf of the University of Leeds, the Institute of Archaeology of London University and Farleigh Dickinson University, New Jersey, I. Field Work and Excavation, Leiden 1974.

LEIGHTON, R. (ed.)

1993 The Protohistoric settlement on the Cittadella (Morgantina Studies IV), Princeton 1993. LEIGHTON, R.

2011 La casa 16W del Bronzo Finale sulla Cittadella di Morgantina (Sicilia): aspetti strutturali, zone di attività e status sociale: Rivista di Scienze preistoriche LXI (2011), pp. 197-214.

LEIGHTON, R. - THOMPSON, S.

2012 Later Prehistoric settlement and the Morgantina Survey: R. LeIGHTON (ed.), Prehistoric Houses at Morgantina. Excavations on the Cittadella of Morgantina in Sicily 1989 - 1994, London 2012, pp. 200-205.

LEVI, S.T.

2010 Dal coccio al vasaio. Manifattura, tecnologia e classificazione della ceramica, Bologna 2010 . 
LONDON, G.A.

2016 Ancient cookware from the Levant. An ethnoarchaeological perspective, Sheffield 2016.

MANSEL, K.

1999 Handgemachte Keramik der Siedlungsschichten des 8 und 7 Jhs. V.Chr. Aus Karthago ein Vorbericht: F. RAKOB (hrsg.), Karthago III, die Deutschen Ausgrabungen in Karthago, Mainz am Rhein 1999, pp. 220-238.

MüLlER, M. (ed.)

2015 Household Studies in Complex Societies. (Micro) Archaeological and Textual Approaches (Oriental Institute Seminar 10), Chicago 2015

Nenci, G. - TusA, S. - TusA, V.

1990 Gli Elimi e l'area elima, fino all'inizio della prima guerra punica: G. NENCI - S. TUSA - V. Tusa (edd.), Atti del Seminario di Studi, Palermo - Contessa Entellina, 25 - 28 Maggio 1989, Palermo 1990

Nicoletti, F. - TUSA, S.

2012 L'età del Bronzo nella Sicilia occidentale: AA.VV. (eds.), Atti della XLI Riunione scientifica. Dai Ciclopi agli Ecisti. Società e territorio nella Sicilia preistorica e

NIGRO, L. protostorica, San Cipirrello, 16 - 19 Novembre 2006, Palermo 2012, pp. 105-130.

2013 Before the Greeks: the Earliest Phoenician Settlement in Motya - Recent Discoveries by Rome «La Sapienza» expedition: Vicino Oriente XVII (2013), pp. 39-74.

2015 Mozia tra VI e V secolo a.C. Monumentalizzazione e organizzazione socio-politica: un nuovo modello: M.P. BAGLIONE - L.M. MichetTi (a cura di), Le lamine d'oro a cinquant'anni dalla scoperta. Dati archeologici su Pyrgi nell'epoca di Thefari e Velians e rapporti con altre realtà del Mediterraneo (Scienze dell'Antichità 21.2), Roma 2015, pp. 225-245.

2016 Mozia nella Preistoria e le rotte levantine: i prodromi della colonizzazione fenicia tra secondo e primo millennio a.C. nei recenti scavi della Sapienza: A. CAZZELLA - A. GUIDI F. Nomi (edd.), Ubi Minor... Le isole minori del Mediterraneo centrale dal Neolitico ai primi contatti coloniali, Convegno di studi in ricordo di Giorgio Buchner, a 100 anni dalla nascita, Anacapri, Capri, Ischia, 27 - 29 Ottobre 2014 (Scienze dell’Antichità 22.2), Roma 2016, pp. 347-370.

2018 La Sapienza a Mozia 2010-2016: il primo insediamento fenicio, l'area sacra di Baal e Astarte, il tofet, la necropoli, l'abitato, i nuovi scavi alle mura - una sintesi: M. GUIRGUIS (ed.), From the Mediterranean to the Atlantic: people, goods and ideas between East and West. II. 8th International Congress of Phoenician and Punic Studies, Italy, Sardinia, Carbonia, Sant'Antioco, 21th-26th October 2013 (Folia Phoenicia 2), Pisa - Roma 2018, pp. 253-277.

NigRo, L. (a cura di)

2004 Mozia - X. Zona C. Il Kothon D. Le pendici occidentali dell'Acropoli. Zona F. La Porta Ovest. Rapporto preliminare della XXII campagna di scavi - 2002 condotta congiuntamente con il Servizio Beni Archeologici della Soprintendenza Regionale per $i$ Beni Culturali e Ambientali di Trapani (Quaderni di Archeologia Fenicio-Punica I), Roma 2004.

2005 Mozia - XI. Il Tempio del Kothon. Rapporto preliminare delle campagne di scavi XXIII e XXIV (2003-2004) condotte congiuntamente con il Servizio Beni Archeologici della Soprintendenza Regionale per i Beni Culturali e Ambientali di Trapani, (Quaderni di Archeologia Fenicio-Punica II), Roma 2005.

2007 Mozia - XII. Zona D, la Casa del sacello domestico, il Basamento meridionale e il sondaggio stratigrafico I. Rapporto preliminare delle campagne di scavi XXIII e XXIV 
(2003-2004) condotte congiuntamente con il Servizio Beni Archeologici della Soprintendenza Regionale per i Beni Culturali e Ambientali di Trapani, (Quaderni di Archeologia Fenicio-Punica II), Roma 2007.

Nigro, L. - SPAgnOLI, F.

2017 Landing on Motya. The Earliest Phoenician Settlement of the 8th century BC and the creation of a West Phoenician Cultural Identity in the excavations of Sapienza University of Rome - 2012-2016 (Quaderni di Archeologia Fenicio-Punica/Colour Monograph 04), Roma 2017.

Niveau, A. - CASTRO, E.

2008 Banquets rituals dans la nécropole punique de Gadir: Food and History 6 (2008), pp. 7-48. NOTARSTEFANO, F.

2012 Ceramica e alimentazione: l'analisi chimica dei residui organici nelle ceramiche applicata ai contesti archeologici, Bari 2012.

OGGIANO, I.

2005 Dal terreno al divino: archeologia del culto nella Palestina del primo millennio, Roma. ORSINGHER, A.

2011 La ceramica punica del IV sec. a.C. dalla Fortezza Occidentale: L. Nigro (ed.), Mozia XIII. Zona F, la Porta Ovest e la Fortezza Occidentale. Rapporto preliminare delle campagne di scavi XXIII e XXVII (2003-2007), condotte congiuntamente con il Servizio Beni Archeologici della Soprintendenza Regionale per i Beni Culturali e Ambientali di Trapani, (Quaderni di Archeologia Fenicio-Punica II), Roma 2011, pp. 112-131.

2013 La ceramica di impasto a Mozia tra cultura fenicia e cultura indigena: L. GIRÒN - M. AZARICH - M. CONÇEICÂO LOPEZ (eds.), Actas del I Congreso Internazionale sobre Estudios Cerámicos, Cadice, 1 - 5 Novembre 2010, Cadice 2013, pp. 757-790.

2018 "Note moziesi” dal tofet: un'analisi a partire dalla sequenza ceramica del campo d'urne: M. GuIRguis (ed.), 8th International Congress of Phoenician and Punic Studies Italy, Sardinia Carbonia, Sant'Antioco 21th-26th October 2013, Folia Phoenicia. An International Journal 2 (2018), pp. 29-34.

ORTON, C. - Tyers, P. - Vince, A.

1993 Pottery in Archaeology. Cambridge Manuals in Archaeology, London 1993.

PEACOCK, D.P.S.

1997 La ceramica romana tra archeologia e etnografia, Bari 1997.

POLANYI, M.

1966 The tacit dimension, New York 1966.

RICE, P.M.

1987 Pottery analysis, Chicago 1987.

RUSSENBERGER, C.

2010 Monte Iato $(\mathrm{Pa})$ : ultime testimonianze di una cultura indigena attorno al 300 a.C.: M. DALLA Riva (ed.), Atti del XVII International Congress of Classical Archaeology Meetings between Cultures in the Ancient Mediterranean, Roma, 22 - 26 Ottobre 2008 (Bollettino di Archeologia online I-2010, Volume Spaciale), Roma 2010, pp. 12-22.

SANTORO Bianchi, S. - Guiducci, G. - TuSA, S.

2003 Pantellerian ware: archeologia subacquea e ceramica da fuoco a Pantelleria, Palermo 2003.

SCHEFFER, C.

1981 Acquarossa. Cooking and cooking stands in Italy 1400 - 400 B.C. (Acta Instituti Romani SEKIK, N. Regni Sueciae IV, XXXVIII:II, 1), Stockholm 1981.

2007 Les potières de Sejnane. Des femmes et un savoir-faire, Tunis 2007. 
2010 À propos du patrimoine immatériel : réflexion autour des savoir-faire des femmes en Tunisie: Quaderns de la Mediterrània 13 (2010), pp. 29-35.

SKIBO, J.

1992 Pottery Function: A Use-Alteration Perspective, New York 1992.

SKIBO, J.- SCHIFFER, M.J.B.

1995 The clay cooking pot: An exploration of women's technology: J.M. SKIBO - W.H. WALKER - A. E. NIELSEN (eds.), Expanding archaeology, Utah 1995, pp. 80-91.

2008 People and Things. A behavioral approach to material culture, New York 2008.

SPAGNOLI, F.

2010 Cooking Pots as an Indicator of Cultural Relations between Levantine Peoples in Late Bronze and Iron Age. Origins, Diffusion and Typological Development of Cooking Ware in Levantine and Cypriot Repertoires (14th-17th centuries B.C.), (Quaderni di Archeologia Fenicio Punica IV), Roma 2010.

SPANÒ GIAMMELLARO, A.

2000 I Fenici in Sicilia: modalità insediamentali e rapporti con l'entroterra: G. PrATs (ed.) Fenicios Y Territorio, Actas del II Seminario Internacional sobre Temas Fenicios, Guardamar del Segura, 9 - 11 Aprile 1999, Alicante 2000, pp. 295-335.

2004a Pappe, vino e pesce salato. Appunti per uno studio della cultura alimentare fenicia e punica: Kokalos 46, (2004), pp. 417-464.

2004b I luoghi della morte: impianti funerari nella Sicilia fenicia e punica, in: A. GoNZÁLEZ PrATs (ed.), El mundo funerario. III Seminario Internacional sobre Temas Fenicios, Guadamar del Segura, 3-5 Maggio 2002, Alicante 2004, pp. 205-251.

SPATAFORA, F.

2000a Indigeni, Punici e Greci in età arcaica e tardo arcaica sulla Montagnola di Marineo nella Valle dell'Eleuterio: A. CorretTi (ed.), Atti delle Terze Giornate Internazionali di Studi sull'Area Elima, Gibellina - Erice - Contessa Entellina, 23 - 26 Ottobre 1997, Pisa Gibellina 2000, pp. 895-918.

2000b La ceramica preistorica dalla "Zona E" dell'abitato di Mozia: A. CORRETTI (ed.), Atti delle Terze Giornate Internazionali di Studi sull'Area Elima, Gibellina - Erice - Contessa Entellina, 23 - 26 Ottobre 1997, Pisa - Gibellina 2000, pp. 919-956.

2010a Indigeni e Greci negli emporia fenici della Sicilia: M. DALLA RIVA (ed.), Meetings between Cultures in the Ancient Mediterranean. Proceedings of the 17th International Congress of Classical Archaeology, Roma, 22 - 26 Settembre 2008 (Bollettino di Archeologia on line I2010, Volume Spaciale), Roma 2010, pp. 34-46.

2010b Per un'«archeologia degli incontri» Sicani ed Elimi nella Sicilia greca: H. TREZINY (ed.), Grecs et Indigénes de la Catalogne à la Mer Noire, Actes des rencontres du programme européen Ramses 2, 2006-2008 (Bibliothéque d'Archéologie Méditeranéenne et Africaine 3), Paris 2010, pp. 25-39.

2012 Interrelazioni e commistioni nella Sicilia nord-occidentale di età arcaica: i contesti funerari come indicatori archeologici: F. BERLINZANI (ed.), Convivenze etniche, scontri e contatti di culture in Sicilia e Magna Grecia (Aristonothos, Scritti per il Mediterraneo antico 7), Trento 2012, pp. 59-90.

SPATAFORA, F. (ed.)

2015 Nutrire la città. A tavola nella Palermo antica. Museo archeologico regionale Antonino Salinas. Palermo 22 Maggio - 30 Novembre 2015, Palermo 2015.

Spatafora, F. - SCIORTINO, G.

2015 Identities under Construction: Sicily in the First Centuries of the First Millennium BCE: G. GARBATI - T. PEDRAZZI (eds.), Transformations and Crisis in the Mediterranean "Identity" and Interculturality in the Levant and Phoenician West during the 12th-8th Centuries BCE, 
Proceedings of the International conference, Rome, 8 - 9 Maggio 2013, Pisa - Roma 2015, pp. 221-229.

Spatafora, F. - VASSALlo, S.

2005 Das Eigene und das Andere. Griechen, Sikaner und Elymer. Neue archaeologische Forschungen im antiken Sizilien. Archäologisch Sammlung der Universität, Ausstellung Zürich, Zurigo, 20 Ottobre 2004 - 28 Febbraio 2005, Palermo - Zurigo 2005.

Spataro, M. - VilLing, A.

$2015 \quad$ Ceramique, Cuisine and Culture, Oxford 2015.

STREIFFERT EIKELAND, K.

2009 Indigenous Households. Transculturation of Sicily and Southern Italy in Archaic period, Göteborg 2009.

TORRES, R.J. - MARAOUNI TELMini, B.

2011 Les sondages dans la zone 2: N. KALlala - J. SANMARTI (eds), Althiburos I. La fouille dans l'aire du capitole et dans la nécropole méridionale, Tarragona 2011, pp. 153-165.

TUSA, S.

1990 Preistoria e Protostoria nel territorio degli Elimi: la genesi di un ethnos e di una cultura: G. NENCI - S. TUSA - V. TUSA (eds.), Gli Elimi e l'area elima. Fino all'inizio della prima guerra punica. Atti del Seminario di Studi, Palermo - Contessa Entellina, 25 - 28 Maggio 1989, $I V, X I V-X V$, Palermo 1990, pp. 31-54.

2008 Gli elementi di interesse paletnologico nella Collezione Whitaker e la Preistoria moziese: R. DE SiMONE - M.P. TOTI (eds.), La collezione Whitaker I, pp. 67-85.

2015 Sicilia Archeologica. I caratteri e i percorsi dell'isola dal Paleolitico all 'età del Bronzo negli orizzonti del Mediterraneo, Roma.

USAI, A.

2013 Spunti di riflessione sull'Età del Ferro della Sardegna: P. An Dommelen - A. Roppa (eds.), Materiali e contesti nell'età del ferro sarda. Atti della giornata di studi, Museo Civico di San Vero Milis (Oristano) 25 Maggio 2012, (Rivista di Studi Fenici 41, 1-2), Roma 2013, pp. 23-34.

VALENTINO, M

2003 La ceramica da fuoco e da cucina: F. SPATAFora (ed.), Monte Maranfusa. Un insediamento nella media Valle del Belice. L'abitato indigeno, Palermo 2003, pp. 255-267.

VAN COMPERNOLLE, $R$.

1983 Femmes indigènes et colonisateurs: Modes de contacts et processus de transformations dans les société anciennes: G. NENCI (ed.), Actes du Colloque de Cortone, 24 - 30 Maggio 1981,

VECCHIO, P. Collection de l'École Française de Rome 67, Roma 1983, pp. 1033-1049.

2013 Morte e società a Mozia. Ipotesi preliminari sulla base della documentazione archeologica della necropoli: H. Hesberg - K.S. Freyberger (eds.), Mitteilungen des Deutschen Archäologischen Instituts Römische Abteilung 119, Berlin 2013, pp. 43-67.

WILK, R.R. - RATHJE, W.L.

1982 Household archaeology: The American Behavioral Scientist 25, 6 (1982), pp. 617-639. 

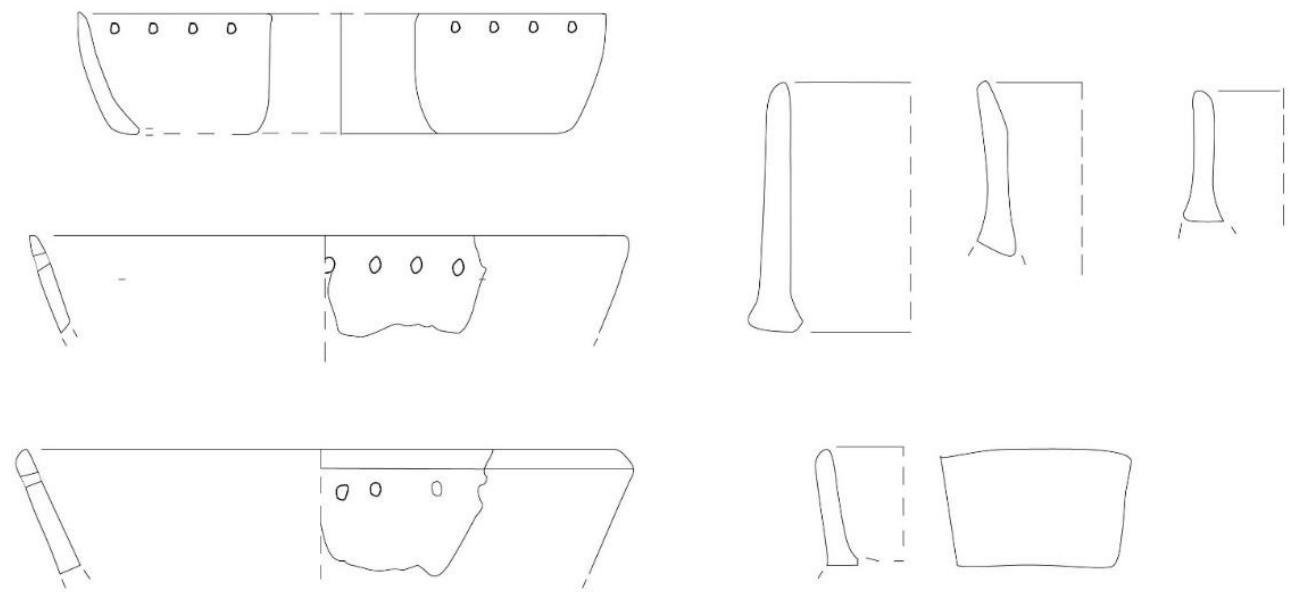

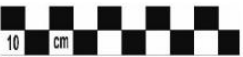

Fig. 1 - Teglie protostoriche dalla Zona E, Mozia (rielaborazione dell'autore da Spatafora 2000b, tav. CLXXX).

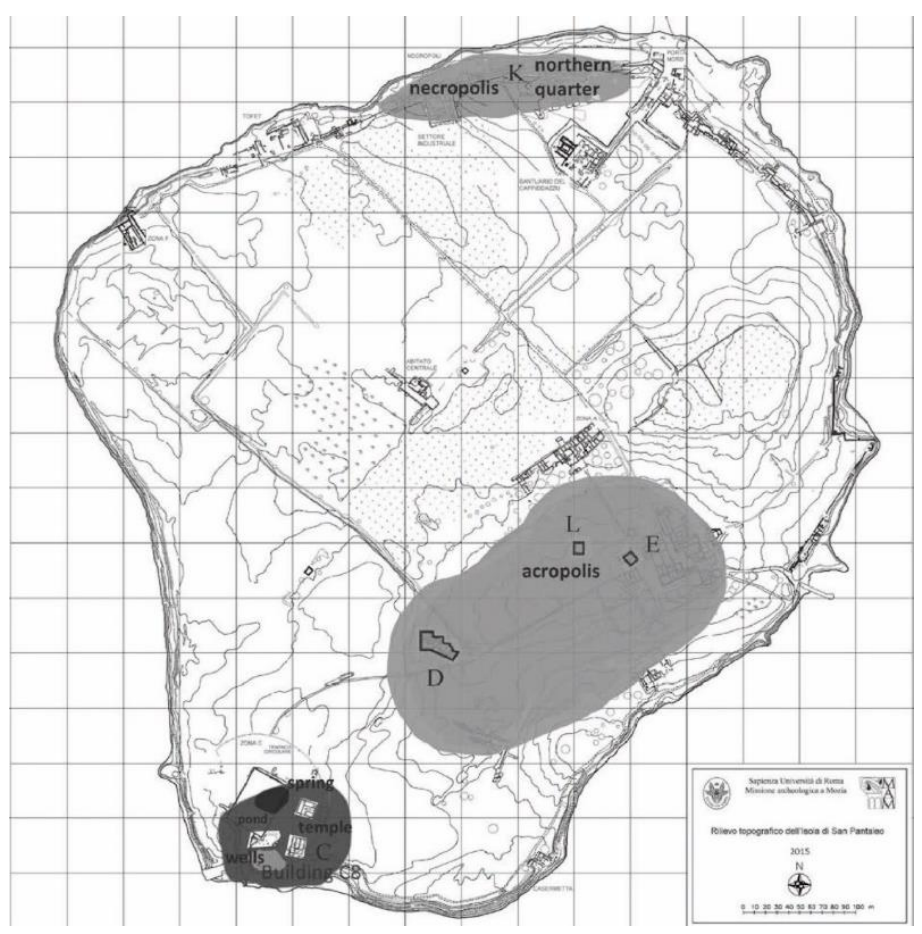

101
Fig. 2 - Carta di Mozia (Nigro 2016, fig. 1). 


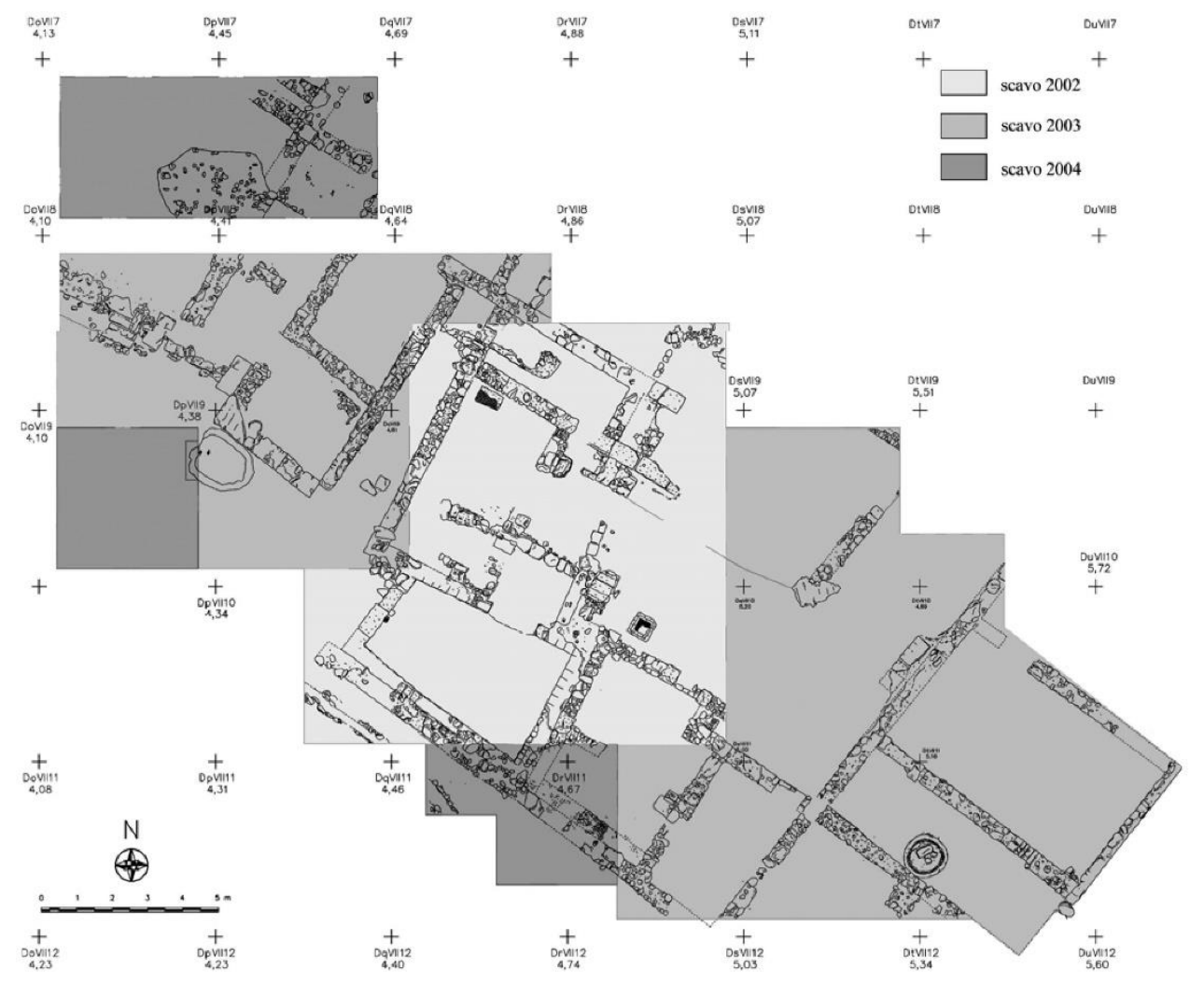

Fig. 3 - Planimetria della Zona D di Mozia (Nigro [a cura di] 2007, 10, fig. 2.1).

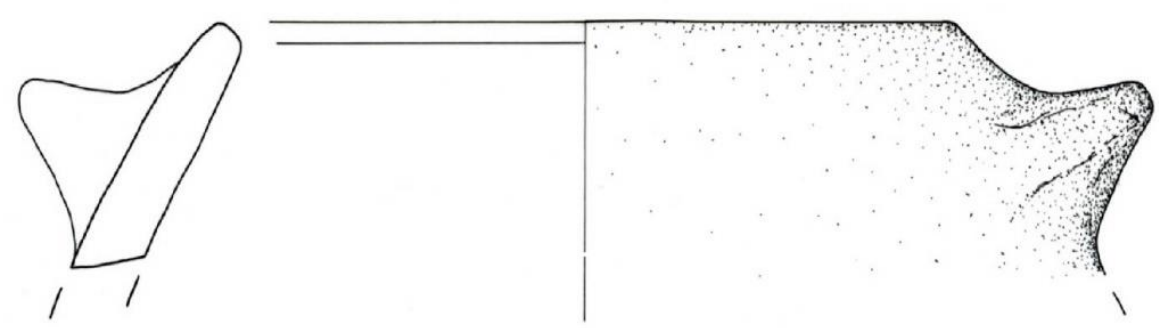

Fig. 4 - Pignatta troncoconica (Caltabiano-Spagnoli 2010, tav. XIII: MD.07.2246/19), scala $1: 2$. 


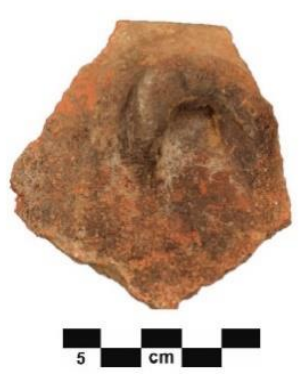

Fig. 5 - Tracce di annerimento sotto la presa MD.07.2246/19 (foto archivio).
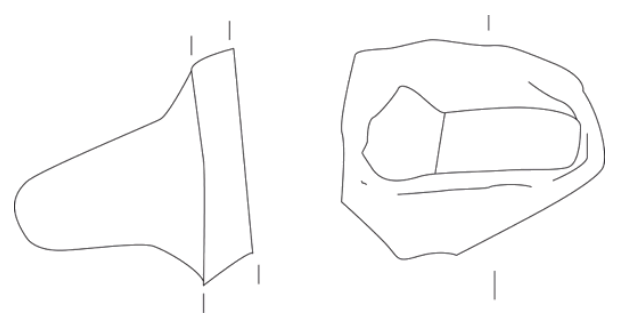

Fig. 6 - Pignatta troncoconica (Nigro [a cura di] 2007, tav. LXXXVII: MD.04.1112/74), scala 1:2.
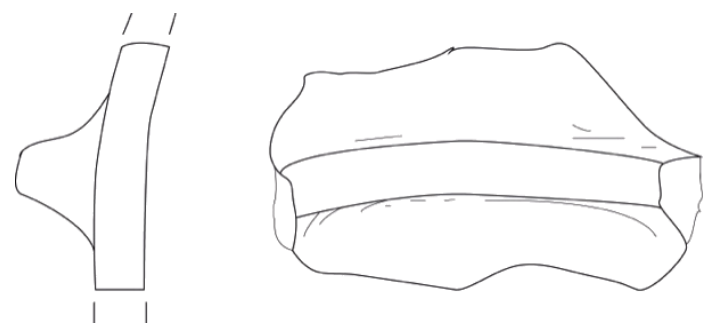

Fig. 7 - Pignatta troncoconica (Nigro [a cura di] 2007, tav. LXXXVII:MD.04.1112/80), scala $1: 2$.

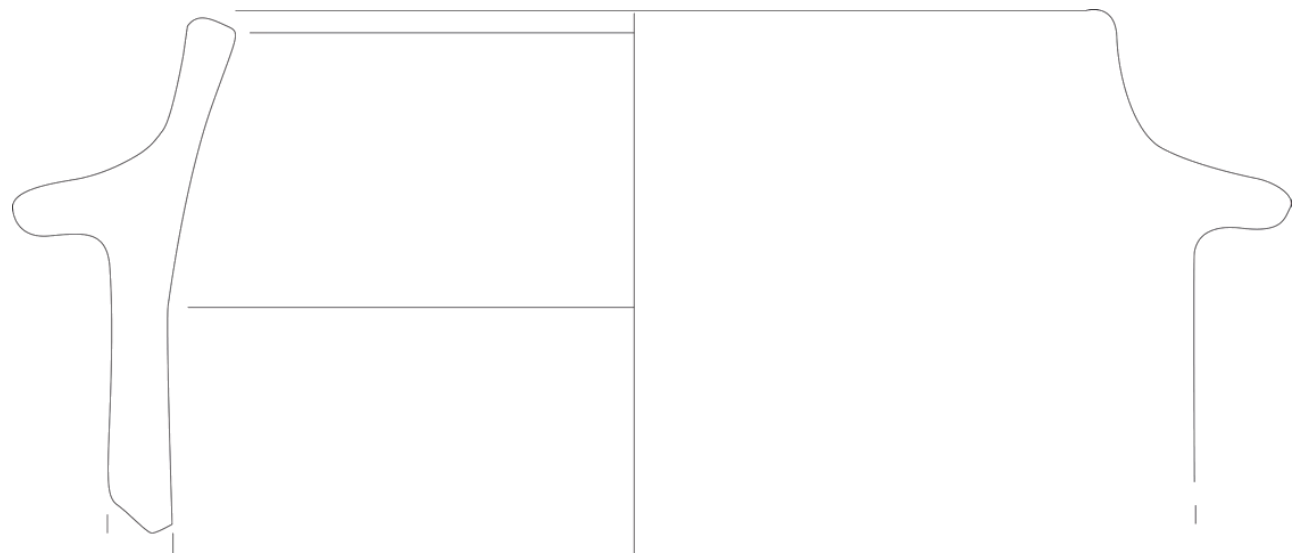

Fig. 8 - Pignatta troncoconica (Nigro [a cura di] 2007, tav. XXXV:MD.03.1009/12), scala $1: 2$. 

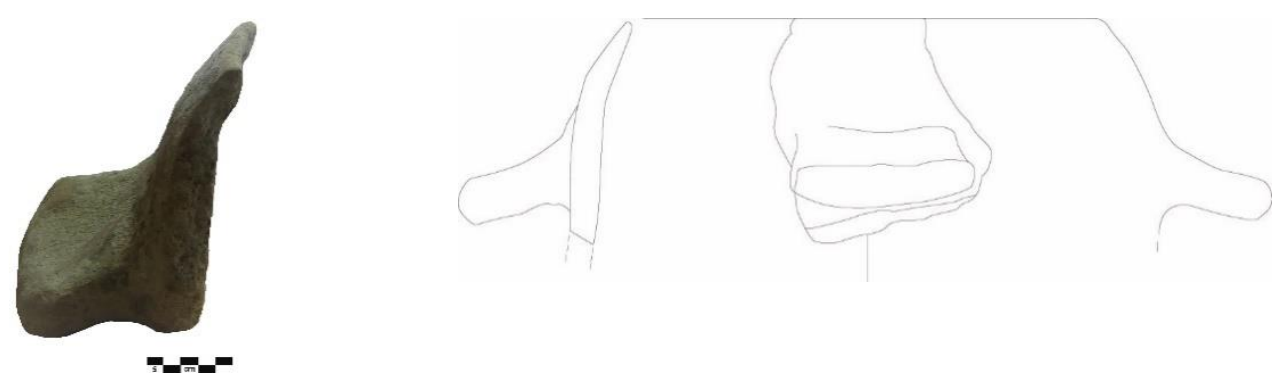

Fig. 9 - Pignatta troncoconica (foto archivio. Disegno in Nigro [a cura di] 2004, tav. LX:MD.02.216/16), scala 1:3.
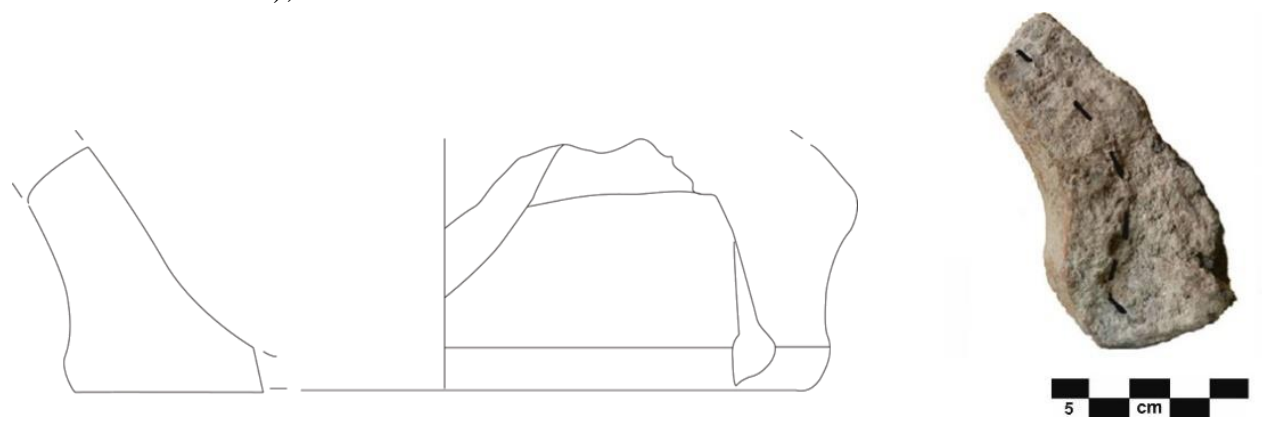

Figg. 10-11 - Teglia MD.02.216/48 (Nigro [a cura di] 2004, tav. LX:MD.02.216/48, scala 1:2) con particolare della sezione (foto archivio).
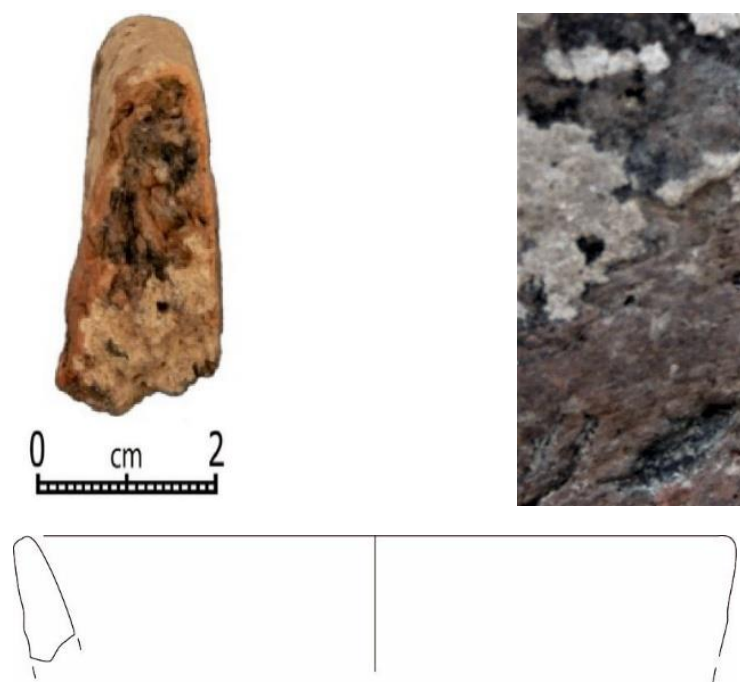

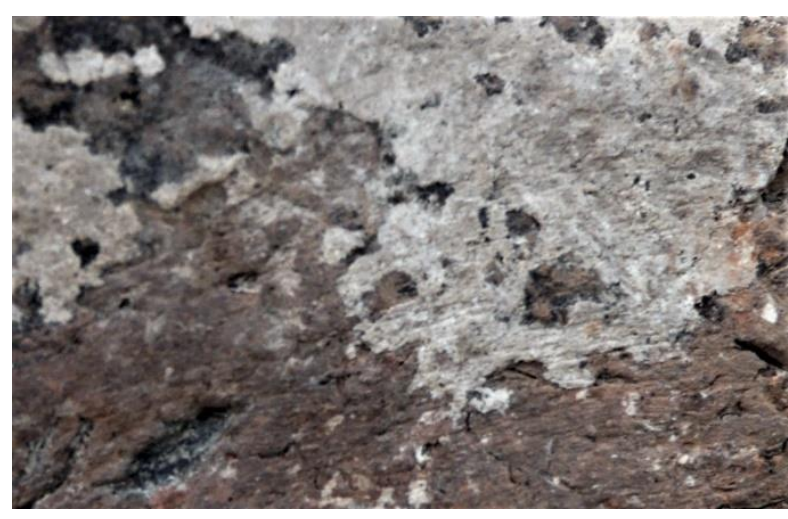

Figg. $\quad$ 12-13 - Teglia MD.04.1090/15 e particolare del trattamento superficiale della lisciatura in senso obliquo sulla stessa teglia (foto archivio. Disegno in Nigro [a cura di] 2007, tav. LXIV:MD.04.1090/15), scala 1:4. 


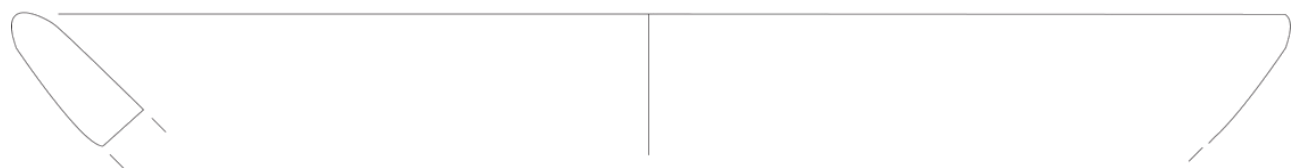

Fig. 14 - Scodellone (Nigro [a cura di] 2007, tav. LV:MD.04.1042/9) scala 1:4.

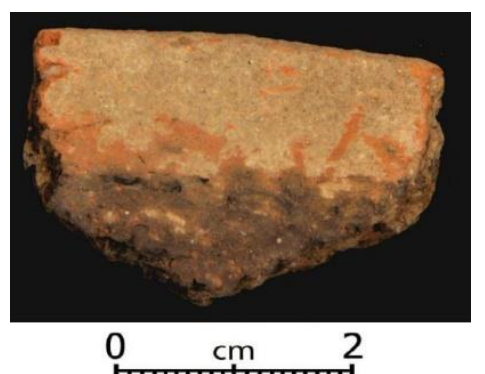

Fig. 15 - Tracce di annerimento su MD.04.1042/9 (foto archivo)
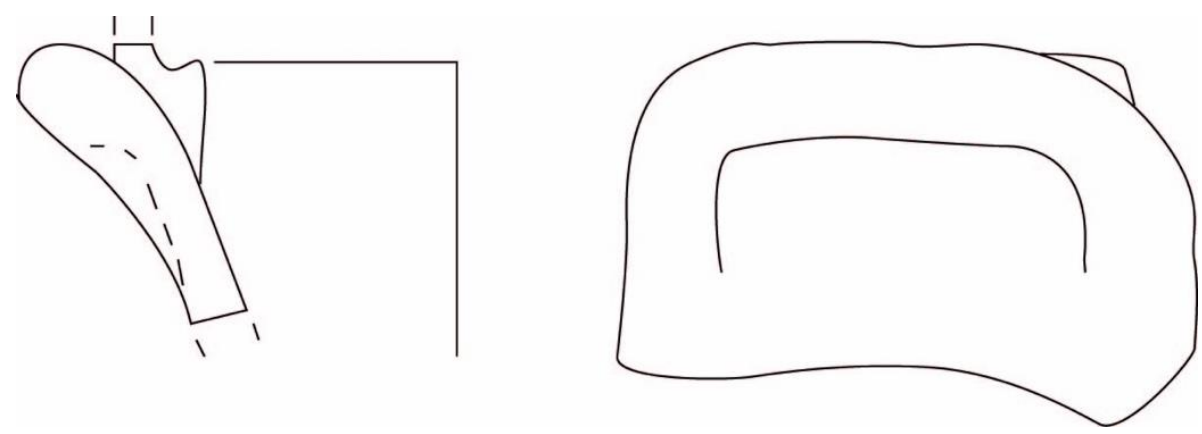

Fig. 16 - Scodellone (Nigro (a cura di) 2007, tav. LIV:MD.03.1042/13), scala 1:1.
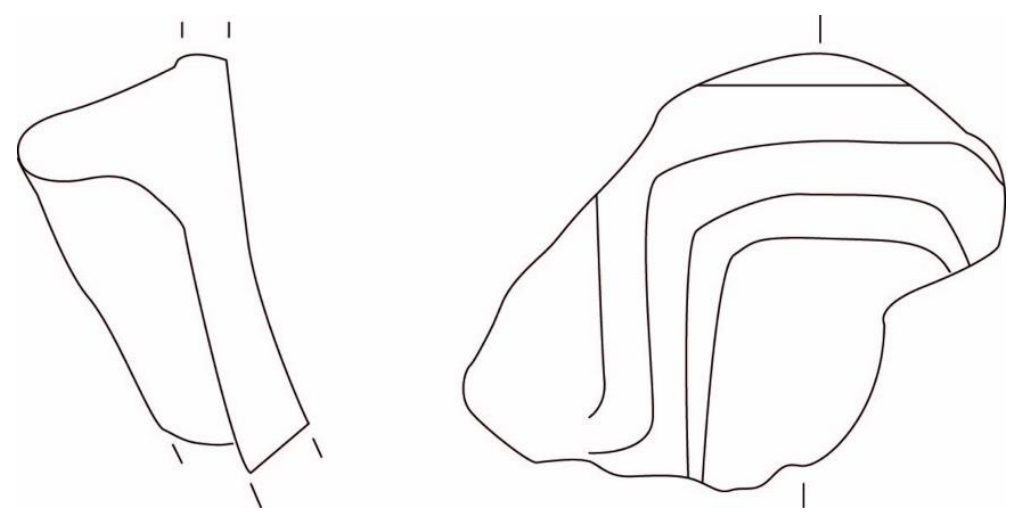

Fig. 17 - Scodellone (Nigro [a cura di] 2005, tav. LXXXVII:MC.04.710/11), scala 1:1. 


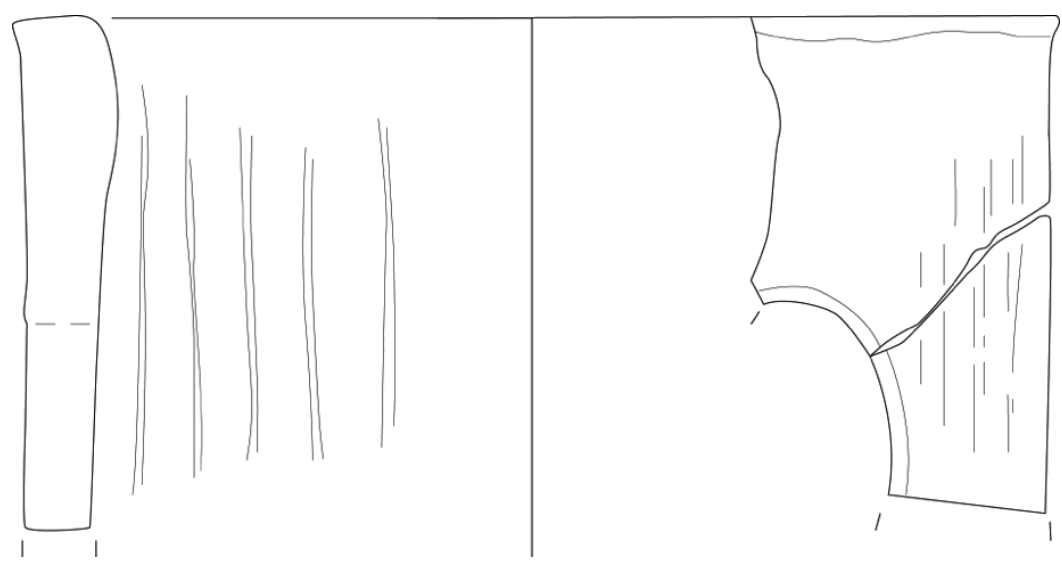

Fig. 18 - Fornello (Nigro [a cura di] 2007, tav. XXX:MD.04.266/8), scala 1:2.

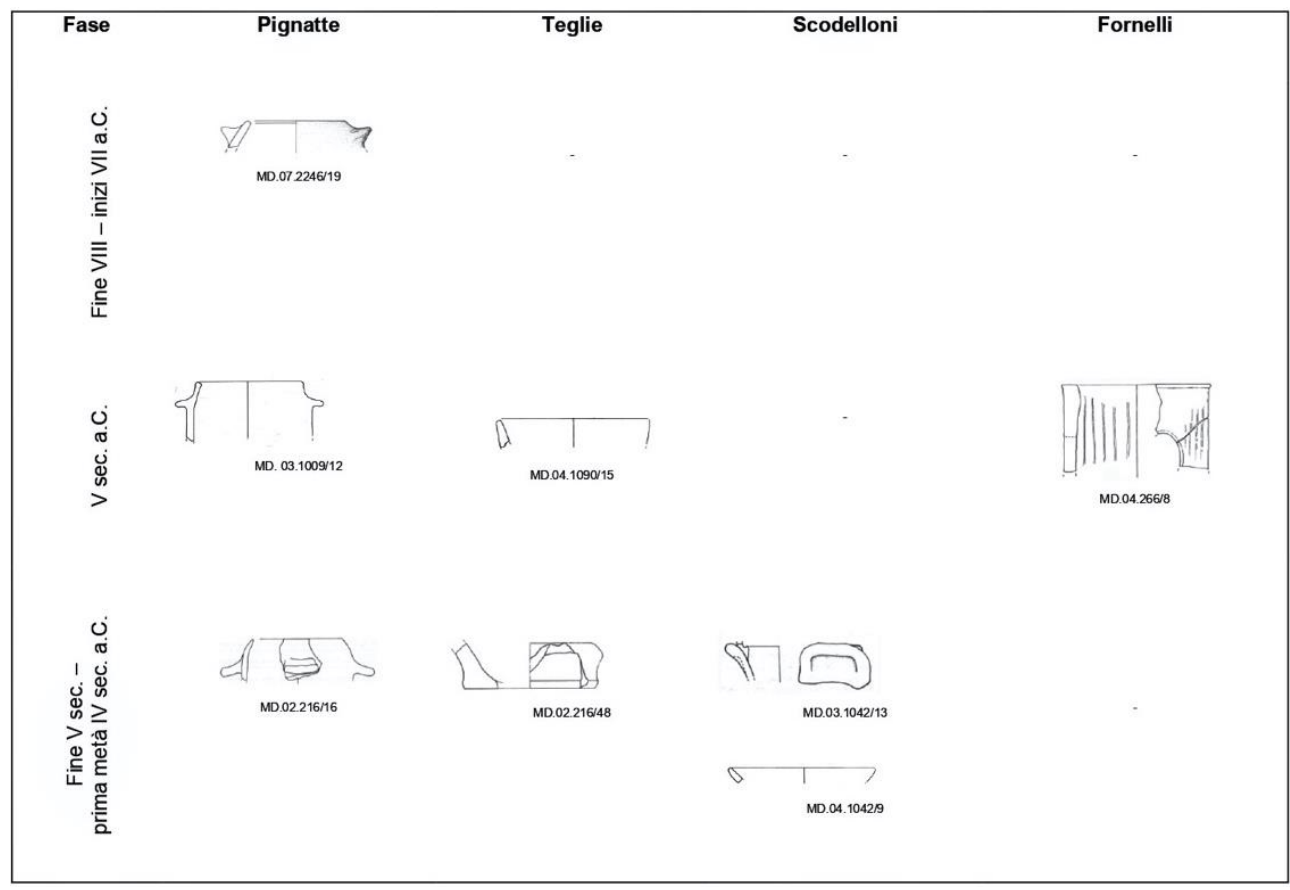

Fig. 19 - Tavola tipologica diacronica delle forme ceramiche d'impasto rinvenute nella Zona D. 


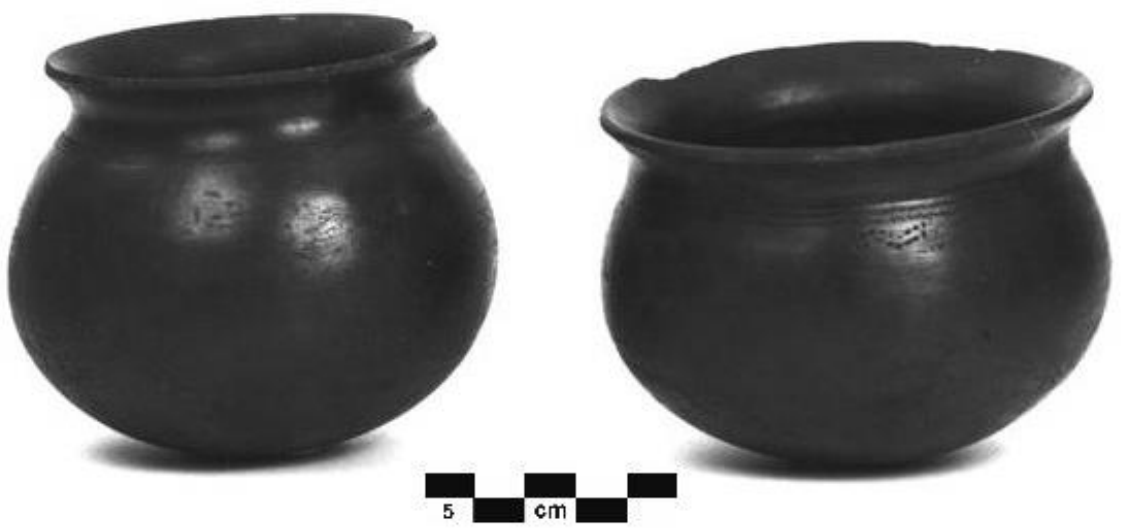

Fig. 20 - Da sinistra: contenitore per la conservazione dell'acqua, olla per la cottura del riso (ittoyom) (rielaborazione dell'autore da Skibo1992, 60, fig. 4.4). 